\title{
Functional Redundancy of Ventral Spinal Locomotor Pathways
}

\author{
David N. Loy, ${ }^{1,2,3,5}$ David S. K. Magnuson,,2,3,5 Y. Ping Zhang, ${ }^{2,5}$ Stephen M. Onifer, $, 2,3,5$ Michael D. Mills, ${ }^{4}$ \\ Qi-lin Cao, ${ }^{2,5}$ Jessica B. Darnall,,2,5 Lily C. Fajardo, ${ }^{4}$ Darlene A. Burke, ${ }^{2,5}$ and Scott R. Whittemore ${ }^{2,3,5}$ \\ ${ }^{1}$ The MD/PhD Program and Departments of ${ }^{2}$ Neurological Surgery, ${ }^{3}$ Anatomical Sciences and Neurobiology, ${ }^{4}$ Radiation \\ Oncology, and ${ }^{5}$ Kentucky Spinal Cord Injury Research Center, University of Louisville School of Medicine, Louisville, \\ Kentucky 40292
}

Identification of long tracts responsible for the initiation of spontaneous locomotion is critical for spinal cord injury (SCl) repair strategies. Pathways derived from the mesencephalic locomotor region and pontomedullary medial reticular formation responsible for fictive locomotion in decerebrate preparations project to the thoracolumbar levels of the spinal cord via reticulospinal axons in the ventrolateral funiculus (VLF). However, white matter regions critical for spontaneous over-ground locomotion remain unclear because cats, monkeys, and humans display varying degrees of locomotor recovery after ventral SCls. We studied the contributions of myelinated tracts in the VLF and ventral columns (VC) to spontaneous over-ground locomotion in the adult rat using demyelinating lesions. Animals received ethidium bromide plus photon irradiation producing discrete demyelinating lesions sufficient to stop axonal conduction in the VLF, VC, VLF-VC, or complete ventral white matter
(CV). Behavior [open-field Basso, Beattie, and Bresnahan (BBB) scores and grid walking] and transcranial magnetic motorevoked potentials (tcMMEP) were studied at 1,2 , and 4 weeks after lesion. VLF lesions resulted in complete loss or severe attenuation of tcMMEPs, with mean BBB scores of 18.0 , and no grid walking deficits. VC lesions produced behavior similar to VLF-lesioned animals but did not significantly affect tcMMEPs. VC-VLF and CV lesions resulted in complete loss of tcMMEP signals with mean BBB scores of 12.7 and 6.5 , respectively. Our data support a diffuse arrangement of axons within the ventral white matter that may comprise a system of multiple descending pathways subserving spontaneous over-ground locomotion in the intact animal.

Key words: locomotion; mesencephalic locomotor region; ventrolateral funiculus; demyelination; spinal cord; rat
In frequently studied rat models of thoracic spinal cord injury (SCI), disruption of ascending and descending white matter tracts is the primary cause for the observed locomotor deficits (Magnuson et al., 1999). Dorsal hemisections, even those including one ventral quadrant, do not induce significant deficits unless they extend ventral to the central canal (Imai and Aoki, 1996; Schwab and Brosamle, 1997). Moreover, thoracic contusion injuries that damage ventral white matter produce severe deficits in hindlimb locomotion (Basso et al., 1996). Together, these data suggest that the initiation of hindlimb locomotor activity is not compromised in the rat unless there is bilateral damage to white matter pathways ventral to the central canal. Current hypotheses concerning the origin of descending locomotor command pathways focus on the mesencephalic locomotor region (MLR), an area in the caudal cuneiform nucleus of mammals that produces locomotor movements when stimulated electrically (Shik et al., 1966, 1967). MLR projections synapse in the pontomedullary medial reticular formation (MedRF) before descending as the reticulospinal tract in the ventrolateral funiculus (VLF) of the spinal cord in which they eventually synapse on central pattern generator (CPG)

\footnotetext{
Received March 22, 2001; revised Sept. 6, 2001; accepted Oct. 3, 2001.

This work was supported by National Institutes of Health Grant NS38665, Norton Healthcare, and the Commonwealth of Kentucky Research Challenge Trust Fund (S.R.W.), the Kentucky Spinal Cord and Head Injury Research Trust (D.S.K.M.), and the Paralysis Project of America (Q.L.C.). We thank Dr. Larry M. Jordan for his critical comments on this manuscript.

Correspondence should be addressed to Dr. Scott R. Whittemore, Kentucky Spinal Cord Injury Research Center, Department of Neurological Surgery, University of Louisville School of Medicine, 511 South Floyd Street, Medical Dental Research Building 616, Louisville, KY 40292. E-mail: swhittemore@louisville.edu. Copyright (ㄷ) 2001 Society for Neuroscience $0270-6474 / 01 / 220315-09 \$ 15.00 / 0$
}

neurons at the lumbar levels (Steeves and Jordan, 1980, 1984; Garcia-Rill et al., 1981; Jordan, 1986, 1991; Noga et al., 1991). Fictive locomotion can also be initiated from the pontomedullary locomotor region (PLR) in the lateral tegmentum of the brainstem via projections to the MedRF, as well as direct projections to the CPG along the dorsolateral funiculus (DLF) white matter tracts (Mori et al., 1977, 1978; Shik and Iagodnitsyn, 1977, 1978; Budakova and Shik, 1980; Kazennikov et al., 1980; Selionov and Shik, 1981). However, lesioning of DLF white matter tracts alone does not prevent MLR- or PLRevoked fictive locomotion (Sholomenko and Steeves, 1987; Noga et al., 1991).

The pathways identified in studies of MLR-induced fictive locomotion suggest that locomotor dysfunction after thoracic SCI may result from damage to reticulospinal fibers projecting from the MedRF to the thoracolumbar spinal cord. The arrangement of thoracic fibers that subserve locomotion has been difficult to ascertain because conventional surgical approaches cannot reliably produce lesions confined to discrete regions of the thoracic ventral spinal cord. These fibers have long been assumed to lie within a relatively discrete region of the VLF based on ventral quadrant cooling experiments (Noga et al., 1991), surgical lesions in the cervical spinal cord (Steeves and Jordan, 1980), and neonatal in vitro studies (Magnuson and Trinder, 1997). However, a growing body of evidence suggests that these specific white matter regions may not represent the full gamut of descending inputs capable of initiating spontaneous locomotion (Sholomenko and Steeves, 1987; Vilensky et al., 1992; Nathan, 1994; Brustein and Rossignol, 1998). The present study was designed to address the 
relative contributions of myelinated fiber tracts located in the adult rat thoracic VLF and ventral columns (VC) to locomotion and to provide anatomical, behavioral, and in vivo electrophysiological correlates for the evaluation of evolving SCI therapies.

\section{MATERIALS AND METHODS}

All surgical interventions and both presurgical and postsurgical care were provided in strict accordance with the Public Health Service Policy on Humane Care and Use of Laboratory Animals, Guide for the Care and Use of Laboratory Animals (Institute of Laboratory Animal Resources, National Research Council, 1996), and with the approval of the University of Louisville Institutional Animal Care and Use Committee.

Radiation. One adult female Fischer 344 rat (175 gm) (Harlan Sprague Dawley, Indianapolis IN) was imaged in a Philips Tomoscan 6000 CT scanner to obtain three-dimensional anatomical information for radiation planning. Slices $(1.5 \mathrm{~mm})$ were obtained using the maximum resolution afforded by the scanner. Radiation planning was undertaken using ADAC Laboratories (Milpitas, CA) Pinnacle treatment planning software running on a Sun Ultra 2 workstation. Photon irradiation was chosen because it optimized the radiation dose along the spinal cord while sparing skin and internal organs.

For the spinal cord lesions, animals were anesthetized with sodium pentobarbital $(50 \mathrm{mg} / \mathrm{kg}$, i.p.) $48 \mathrm{hr}$ after ethidium bromide (EB) injections, as described below, and secured in the prone position. Animals were then shielded with custom cerrobend collimators that localized the radiation dose to a $3 \mathrm{~cm}$ length of cord restricted to the dorsal surface. 40Gy (Blakemore and Patterson, 1978) were delivered to the spinal cord using two Elekta SL-25 linear accelerators delivering $6 \mathrm{MV}$ lateral opposed photon fields at a $100 \mathrm{~cm}$ source to axis distance. The CT scanners and linear accelerators are housed at the James Graham Brown Cancer Center (University of Louisville). After irradiation, animals were given gentamycin $(0.03 \mathrm{mg} / \mathrm{kg}$, i.m. $)$ and a $10 \mathrm{cc}$ subcutaneous bolus of lactated Ringer's solution, and their cages were placed on a $37^{\circ} \mathrm{C}$ heating pad overnight.

Surgery. Thirty-two adult female Fischer 344 rats (158-179 gm) were anesthetized with sodium pentobarbital $(50 \mathrm{mg} / \mathrm{kg}$, i.p.) and subsequently given prophylactic intramuscular injections of gentamycin $(0.03$ $\mathrm{mg} / \mathrm{kg}$, i.m.). After dorsal laminectomies at the T9 and T10 vertebrae, the dura was opened transversely at three longitudinal sites $2 \mathrm{~mm}$ apart. Bilateral injections $(0.5 \mu \mathrm{l}$ per injection) of $0.3 \mathrm{mg} / \mathrm{ml} \mathrm{EB}$ were placed at stereotaxic coordinates within the VC $(0.3 \mathrm{~mm}$ lateral to midline at depths of $2.0 \mathrm{~mm}$ and then $1.7 \mathrm{~mm}), \mathrm{VLF}(0.7 \mathrm{~mm}$ lateral to midline at depths of $1.5 \mathrm{~mm}$ and then $1.2 \mathrm{~mm}$ ), combined VC-VLF, and CV (combined VC-V LF with an additional injection $0.7 \mathrm{~mm}$ lateral to midline at $1.1 \mathrm{~mm}$ ) using custom-pulled glass micropipettes (20 $\mu \mathrm{m}$ internal diameter) attached to a Parker picospritzer (Magnuson et al., 1999; Cao et al., 2001). Each injection was delivered over $10 \mathrm{sec}$ and allowed to diff use for $3 \mathrm{~min}$ before micropipette movement. The surgical site was irrigated copiously with warmed normal saline and closed in layers using Vicryl 5-0 sutures. One control group $(n=4)$ received injections of normal saline at coordinates identical to the CV lesion.

Spinal cord processing. Control and injured rats were similarly processed for histological examination at 2 and 4 weeks after injury. The histological results were similar at 2 and 4 weeks, and only the 4 week data will be presented. All animals were deeply anesthetized with sodium pentobarbital (60 mg/kg, i.p.) and perfused transcardially with $100 \mathrm{ml}$ of cold $0.1 \mathrm{M}$ PBS, followed by $400 \mathrm{ml}$ of $4 \%$ paraformaldehyde in $0.1 \mathrm{M}$ phosphate buffer, $\mathrm{pH}$ 7.4. Spinal cords were post-fixed at $4^{\circ} \mathrm{C}$ overnight and then transferred to $30 \%$ sucrose at $4^{\circ} \mathrm{C}$ for cryoprotection $(48 \mathrm{hr})$. Afterward, spinal cords were mounted in TBS compound (Triangle Biomedical Sciences, Durham, NC) and sectioned at $16 \mu \mathrm{m}$ with a cryostat. Myelin staining was performed with standard luxol fast blue methods. Immunostaining of axons was performed as described previously (Cao et al., 2001) using $160 \mathrm{kDa}$ neurofilament (NF-M) (1:200; Chemicon, Temecula, CA). Images were captured using a Zeiss (Oberkochen, Germany) Axioskop 50 upright microscope equipped with an Optronix three-chip CCD camera connected to a Power Macintosh 9600 computer (Apple Computers, Cupertino, CA) running NIH Image software.

Functional outcome measures. As an in vivo electrophysiological measure of motor pathway function, transcranial magnetic motor evoked potentials (tcMMEP) were performed at 1, 2, and 4 weeks after lesion, using methods described previously (Magnuson et al., 1999). Briefly, the tcMMEP relies on the activation of subcortical structures with an elec- tromagnetic coil placed over the cranium. Action potentials descend in the ventral spinal cord and synapse onto motoneuron pools in which output signals can be recorded from both of the gastrocnemius muscles. tcMMEP latency measurements have been shown to be very consistent in comparisons made between animals (Linden et al., 1999; Magnuson et al., 1999).

The Basso, Beattie, and Bresnahan (BBB) scale, a 22-point scale $(0-21)$ that is based on the recovery of hindlimb function after contusive thoracic spinal cord injuries in rats (Basso et al., 1995), was used for the evaluation of spontaneous open-field locomotion in all lesioned animals. During the evaluation, animals were allowed to walk freely on the open-field surface for 4 min while being observed by two scorers lacking knowledge of the experimental groups.

Grid walking was also used to assess hindlimb locomotor deficits (Behrmann et al., 1992). Animals underwent $5 \mathrm{~d}$ of acclimation on the grid-walking apparatus while also being exposed to food incentives. After acclimation, animals were food deprived for $2 \mathrm{~d}$, followed by a diet restricted to $67 \%$ of normal intake. Animals were always allowed access to water ad libitum. Animals were trained for $5 \mathrm{~d}$ to cross a $150 \mathrm{~cm}$ concourse containing $4 \mathrm{~cm}$ square holes for food rewards. The gridwalking surface was covered by a $10 \times 10 \times 140 \mathrm{~cm}$ wooden tunnel with a clear Plexiglas top leading to a darkened box at the end that contained food rewards. Body weights were maintained at $90 \%$ of pretest values while animals underwent three prelesion testing sessions with $1 \mathrm{~d}$ between each session. Total hindlimb footfalls were counted by two observers unaware of the experimental groups during each trial. A footfall was defined as a misplacement such that the hindpaw to ankle fell completely below the level of the grid. Before surgery, animals were allowed to return to their pretraining weights with ad libitum food. Testing was resumed without food restriction once weekly at 1,2 , and 4 weeks after lesion.

Statistical analyses. Repeated-measures ANOVAs were used to analyze BBB scores for all groups and electrophysiologic measures in the VC group. One-way ANOVAs were used to analyze differences in BBB scores and electrophysiologic measures among groups at each time point. Repeated-measures and one-way ANOVAs were followed by Tukey's or Student-Newman-Keuls post hoc $t$ tests as appropriate for sample size in significance analyses. Student's $t$ tests were used (for equal and unequal variances as applicable) to analyze time points of CV control and VLF groups with respect to VC prelesion values for tcMMEP latencies and amplitudes.

\section{RESULTS}

To address the relative contributions of thoracic ventral spinal myelinated tracts to locomotion, well characterized EB combined with $\mathrm{x}$-irradiation (EB-X) methods were used to create discrete bilateral demyelinating lesions in the VLF and VC (Honmou et al., 1996; Jeffery et al., 1999). Unlike previously used mechanical methods, these lesions provided high specificity without significant manipulation of other regions of the spinal cord. tcMMEPs were monitored as an in vivo functional measurement because these signals are severely affected by standardized thoracic contusions that produce locomotor deficits. The goal of localizing this signal was to establish a reliable pathway for therapies that could be monitored for efficacy in vivo.

\section{Demyelination}

Figure $1 B-E$ shows representative luxol fast blue-stained transverse sections of T10 spinal cord 4 weeks after irradiation and bilateral injections of EB (Blakemore and Patterson, 1978; Honmou et al., 1996) into the VLF $(B)$, VC $(C)$, combined VLF-VC $(D)$, or $\mathrm{CV}(E)$. Control animals $(n=4)$ received injections of $0.9 \%$ normal saline into stereotaxic coordinates identical to the CV lesion (Fig. 1A). Spinal cords from the control group did not show evidence of demyelination and were structurally intact, aside from one animal that exhibited a slight expansion of the central canal. Histological examination of VLF-lesioned spinal cords $(n=12)$ revealed well defined areas of demyelination that consistently followed a linear diffusion pattern extending away from each injection point toward the central canal and peripher- 


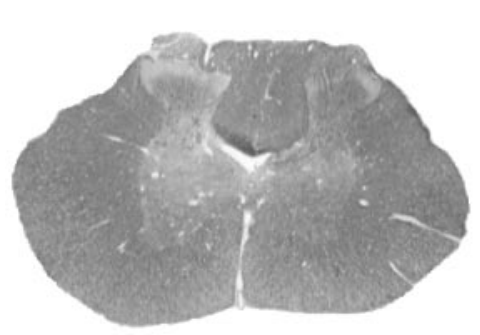

A

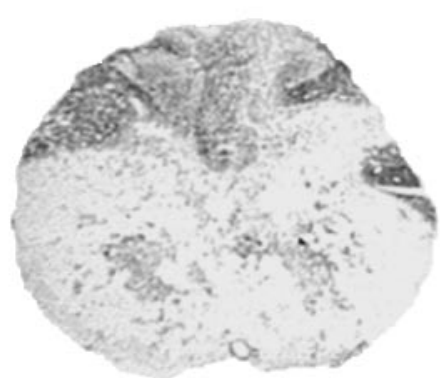

E

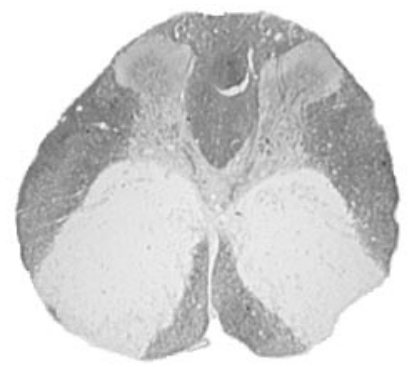

B

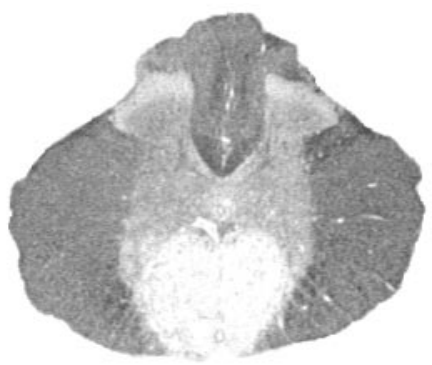

C

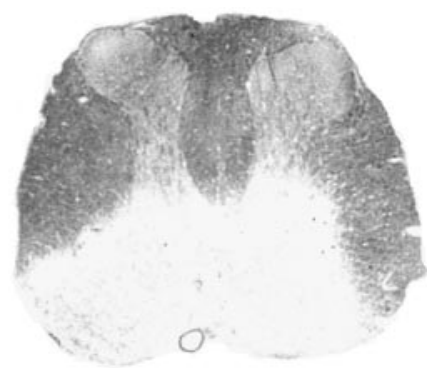

D

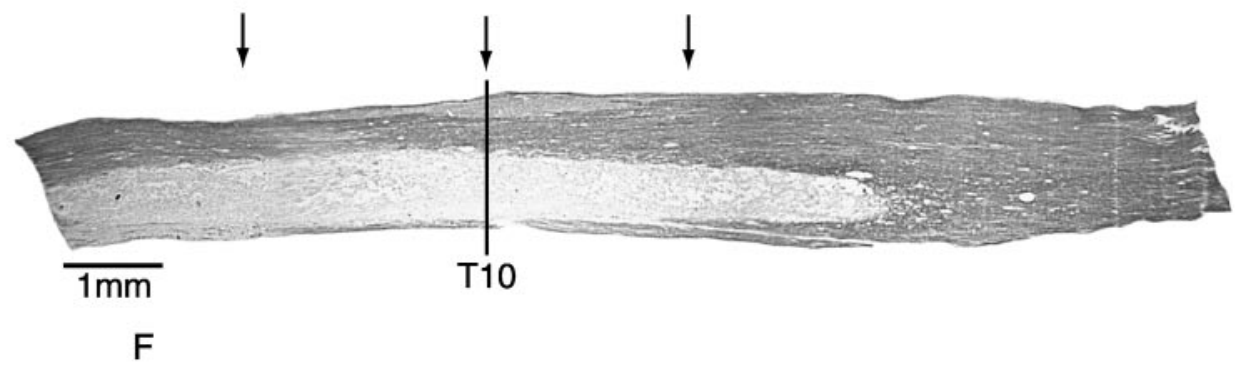

Figure 1. Representative luxol fast blue-stained transverse sections of T10 (injury epicenter) spinal cord taken from representative animals in each group 4 weeks after the indicated lesions. $A, \mathrm{CV}$ saline control; $B, \mathrm{VLF} ; C, \mathrm{VC} ; D$, VLF-VC; $E, \mathrm{CV}$. Lesions for all animals in the respective groups shared equivalent histopathology. $F$, Representative parasagittal section taken from the epicenter of a VLF-lesioned animal. Demyelinated lengths of axons $(6-8 \mathrm{~mm})$ were sufficient to stop axonal conduction in all lesion types. Arrows indicate the sites of EB injections. Similar histopatholgy was observed in all VLF animals that were similarly sectioned.

ally to the dura edge (Fig. $1 B$ ). The central extent of VLF lesions exhibited varying degrees of ventral gray matter involvement that frequently encompassed laminas VII, VIII, and IX. It should be noted that the gray matter component of thoracic lesions produced in this study should not have contributed to the observed results because Magnuson et al. (1999) previously demonstrated no significant effect on locomotor behaviors or tcMMEP recordings after selective thoracic gray matter lesions. Widths of the demyelinated bands ranged from 0.8 to $1.0 \mathrm{~mm}$ within the VLF, and sparing of triangular regions of ventral white matter was observed both lateral to the lesion borders and medially within the ventral columns.

Figure $1 F$ shows a parasagittal section taken from a representative VLF spinal cord 4 weeks after lesion induction. It demonstrates the distribution of demyelination reproducibly seen between the three injection sites (arrows). The rostrocaudal extent of demyelination in all lesions ranged from 6 to 8 $\mathrm{mm}$, lengths sufficient to prevent axonal conduction in normally myelinated axons (Honmou et al., 1996), and did not extend rostrally beyond T8 or caudally beyond T11. Animals exhibiting demyelination in the region of the lateral funiculus within the ventral spinal cord normally spared by VLF lesioning did not exhibit significant locomotor deficits $(n=2$; data not shown).

VC lesions $(n=7)$ displayed similarly well demarcated areas of demyelination that extended laterally through the ventral columns from the ventromedial fissure to involve the medial aspects of the ventral gray matter (Fig. 1C). Demyelination of white matter dorsal to the central canal was not observed in VC lesions. Demyelination was restricted to a $0.1-0.2 \mathrm{~mm}$ area width immediately adjacent to the lateral gray matter in the largest VLF and combined VLF-VC lesions. VLF-VC lesions $(n=6)$ spared only the aspects of ventral white matter dorsolateral to VLF lesion borders (Fig. $1 D)$. CV lesions $(n=3)$ covered an extensive area of ventral white and gray matter because the aim was to create a functional ventral hemisection while leaving the dorsal funiculus and dorsolateral funiculi undisturbed (Fig. 1E). The volumes of EB needed to produce CV lesions proved more difficult to control surgically, producing some partial demyelination dorsal to the desired lesion area.

Axonal densities appeared to be reduced within areas of demyelination at 4 weeks as described previously in EB-X adult rat thoracic dorsal column lesions (Jeffery et al., 1999); however, axons that remained were intact because they did not exhibit end-bulb formation (Fig. 2). No differentiation between demyelinated and unmyelinated axons was attempted; therefore, damage to unmyelinated axons could not be ruled out. Macrophages were abundant within all lesions at 4 weeks (data not shown), consistent with previous results (Blakemore and Patterson, 1978; Honmou et al., 1996). During the 4 week course of the study, lesions should not have undergone spontaneous remyelination after 40Gy doses of irradiation (Blakemore and Patterson, 1978). Present evidence of the lack of spontaneous remyelination included lesion epicenters devoid of luxol fast blue staining at 4 weeks, persistent absence of tcMMEPs or stable tcMMEP latency measurements in VLF- and VLF-VC-lesioned animals, and the observed lack of recovery of BBB scores in VLF-VCand CV-lesioned animals (described below).

\section{tcMMEP responses}

tcMMEP responses were recorded from the left and right gastrocnemius muscles before lesion and 1, 2, and 4 weeks after lesion. Data from control animals are presented in Table 1 and Figure $3 A$. Animals receiving VLF lesions either displayed no tcMMEP response (VLFb) or severely impaired responses (VLFa) to transcranial magnetic evoked stimulation at 1 week 


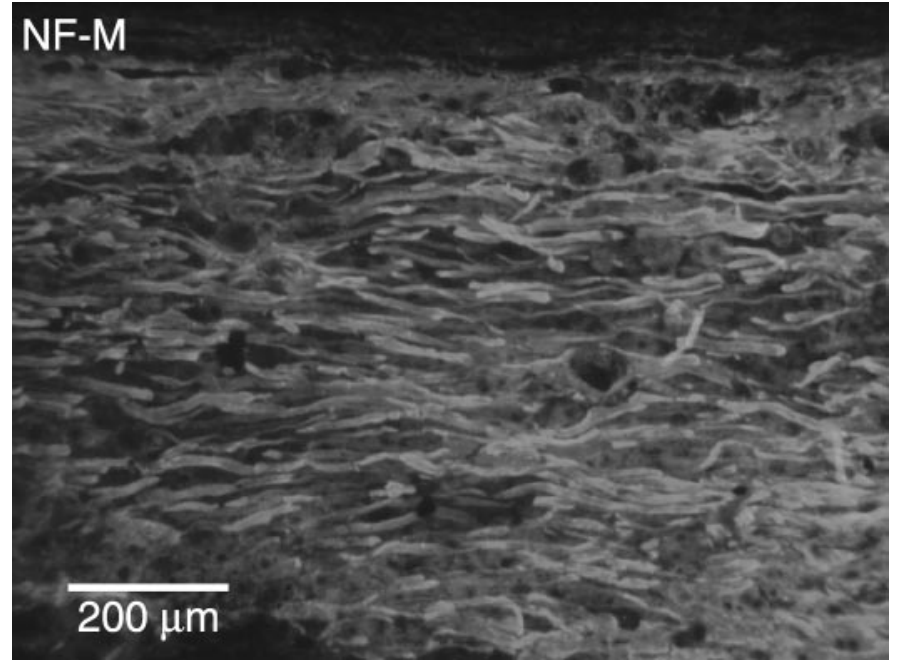

Figure 2. Axons within the demyelinated VLF lesion epicenter. NF-M immunostaining did not demonstrate end-bulb formation in axons remaining within the lesion epicenter at 4 weeks. Similar data were seen in all VLF animals that were similarly immunostained.

after lesion (Table 1; Fig. 3B,C). It is worth noting that two of seven VLFb animals that did not have tcMMEP responses at week 1 regained grossly delayed responses by week 2 that remained unchanged to the end of the study despite any histologic evidence of remyelination. In these two animals, small areas of incompletely demyelinated axons may have experienced a transient period of complete dysfunction that recovered by week 2 to a functional level limited by the extent of damage to the myelin sheath. tcMMEP responses with increased latencies and severely reduced amplitudes were seen in VLFa rats (Table 1; Figs. 3C, $4 D-F)$. Histologic analyses of these animals consistently demonstrated either slight axial rotational errors in the stereotaxic placement of EB or volumetric errors that left small regions of patchy myelination along the borders of the tcMMEP fiber strip (Fig. $4 D-F$ ). Figure $4 A$ illustrates the hypothetical pathway responsible for tcMMEP conduction in the thoracic ventral white matter. Sections of lesion epicenters from rats with no (Fig. 4B) or severely attenuated tcMMEP responses (Fig. $4 D-F$ ) were overlaid onto lesions that did not significantly affect tcMMEPs (Fig. $4 C$ ) to delineate the minimal bilateral areas involved in tcMMEP conduction.

VC-lesioned animals did not display significant changes in tcMMEP responses compared with saline controls, although latencies were slightly delayed in both groups compared with prelesion values (Table 1; Fig. 3D). VLF-VC- and CV-lesioned animals never displayed tcMMEP responses after lesion induction (Table1; Fig. $3 E$ ), because the tcMMEP fiber strip was always located well within the lesion borders (Fig. 4).

\section{Locomotor deficits}

$\mathrm{BBB}$ open-field locomotor scores for right and left hindlimbs were assessed before lesion and 1,2, and 4 weeks after lesion. Paired $t$ test analyses did not demonstrate statistical differences $(p=0.35)$ between the scores for the limbs of each rat, and thus they were averaged. Table 1 shows the mean \pm SD BBB scores for all groups of rats. Control animals (Fig. $5 E$ ) demonstrated $\mathrm{BBB}$ scores throughout the 4 weeks, which were not significantly different from those of naïve rats (BBB score of 21). The mean BBB scores for VC-lesioned animals $(18.5 \pm 1.0$ at 4 weeks) (Fig. $5 C, D$ ) indicate modest but significant reductions in BBB scores compared with pretest values and controls. VLF-lesioned animals (Fig. 5A,B) scored similarly to VClesioned animals despite the absence of (Fig. 3B; Table 1, $\mathrm{VLFb}$ ) or severe deficits in (Fig. 3C; Table 1, V LFa) tcMMEP responses (BBB scores of $17.0 \pm 0$ and $18.0 \pm 1.2$, respectively). Two-way ANOVAs of both VLF and VC groups showed no significant recovery of hindlimb locomotor function by 4 weeks. VLF-VC-lesioned animals (Fig. $5 F$ ) exhibited weightsupported locomotion with pronounced forelimb-hindlimb coordination deficits (BBB score of $12.7 \pm 0.8$ at 4 weeks). $\mathrm{CV}$-lesioned animals (Fig. $5 G$ ) displayed severe locomotor deficits (BBB score of $6.5 \pm 2.1$ at 4 weeks) and were unable to support weight with the hindlimbs throughout the course of the study. The paralysis associated with CV-lesioned animals was flaccid, with passive flexion-extension maneuvers revealing no evidence of spasticity in the large hindlimb muscle groups.

BBB scores were not assigned to animals during the first $6 \mathrm{~d}$ after surgery. However, VLF- and VC-lesioned animals did not exhibit loss of weight support during this interval after lesioning when they were removed from their cages and placed on a flat surface during twice daily physical exams. VLF-VC- and CV-lesioned animals exhibited obvious severe locomotor deficits or paraplegia within 48-72 hr after EB injections that did not resolve at any time before the postlesion week $1 \mathrm{BBB}$ scoring session.

It is important to note that the discrete nature of the VLF and VC lesions produced locomotor behavioral changes that were not accurately represented by the BBB scoring system. To evaluate these behavioral changes for both VLF and VC groups, two observers lacking knowledge of the experimental groups recorded spontaneous locomotor behaviors not described in the $\mathrm{BBB}$ scoring criteria that are not normally present in naïve animals. Figure 6 represents the most frequent hindlimb behaviors. All VLF (VLFa and VLFb)-lesioned animals $(n=12)$ were observed to always walk on their toes in severe plantar flexion without heel contact on the walking surface (Fig. $5 A, B$ ). Toe walking was never seen in control rats. Passive flexion-extension maneuvers of the hindlimbs again did not uncover any evidence of spasticity in the large muscle groups. The base of the tail and ridge of the lumbosacral spine were also noticeably elevated during open-field locomotion in VLF-lesioned animals. Toe walking was also observed in $53 \%$ of VC-lesioned animals $(n=$ 7); however, the degree of plantar flexion was not as qualitatively severe as that of the VLF group because their heel frequently made contact with the open-field surface (Fig. 5C,D). Sixty-seven percent of VC-lesioned animals and $43 \%$ of VLF-lesioned animals also displayed a phenomenon of heavy stepping during locomotion that can be defined as an audible noise associated with the placement of each hindpaw onto the open-field testing surface. This is in contrast to normal animals that move almost silently, even during brisk locomotion.

Other less frequently observed behaviors included the following: 3 of 12 VLF-lesioned animals that had a tendency to take small shuffle-like steps, one VC-lesioned animal of seven that preferentially moved by stepping laterally, and one VC-lesioned animal that converted to an abnormally wide stance after coming to a halt. Histological analysis of the VC-lesioned animal with a widened stance revealed demyelination in the ventral columns that extended into the lumbar enlargement, and tcMMEP responses were comparable with controls. This animal was excluded from the study. No histological or electrophysiological correlates 


\begin{tabular}{|c|c|c|c|c|}
\hline & Before lesion & 1 week after lesion & 2 weeks after lesion & 4 weeks after lesion \\
\hline \multicolumn{5}{|l|}{ BBB scores } \\
\hline $\mathrm{CV}$ saline control & $21(4)$ & $20.5 \pm 0.6(4)^{\dagger}$ & $19.0 \pm 1.2(4)^{* \dagger}$ & $20.5 \pm 0.6(2)^{\dagger}$ \\
\hline $\mathrm{VC}$ & $21(10)$ & $17.0 \pm 0.8(7)^{*+\dagger}$ & $17.9 \pm 1.2(7)^{* \dagger}$ & $18.5 \pm 1.0(6)^{* \dagger \delta}$ \\
\hline VLFa & $21(12)$ & $17.0 \pm 0.6(5)^{*+\dagger}$ & $17.7 \pm 0.8(7)^{* \dagger}$ & $18.0 \pm 1.2(5)^{* \dagger \dagger \delta}$ \\
\hline $\mathrm{VLFb}$ & & $16.0 \pm 1.7(7)^{* \dagger \dagger}$ & $17.2 \pm 0.4(5)^{* \dagger}$ & $17.0 \pm 0.0(2)^{*+\dagger \delta}$ \\
\hline VLF-VC & $21(6)$ & $11.5 \pm 0.7(6)^{*+\dagger \dagger}$ & $12.6 \pm 0.8(6)^{* \dagger \dagger}$ & $12.7 \pm 0.8(6)^{*+\dagger \dagger}$ \\
\hline $\mathrm{CV}$ & $21(3)$ & $4.8 \pm 5.4(3)^{*+\dagger \dagger \dagger}$ & $4.1 \pm 3.1(3)^{* \dagger \dagger \dagger}$ & $6.5 \pm 2.1(2)^{*+\dagger \dagger}$ \\
\hline \multicolumn{5}{|c|}{ tcMMEP latency (msec) } \\
\hline CV saline control & & $6.16 \pm 0.07(4)^{\bullet \dagger}$ & $6.11 \pm 0.16(4)^{\bullet \dagger}$ & $6.01 \pm 0.33(2)^{\dagger}$ \\
\hline VC & $5.74 \pm 0.14(10)$ & $6.06 \pm 0.15(7)^{* \dagger}$ & $6.01 \pm 0.23(7)^{* \dagger}$ & $5.97 \pm 0.13(6)^{* \dagger}$ \\
\hline VLFa & & $6.57 \pm 0.11(5)^{\bullet \dagger \dagger}$ & $6.69 \pm 0.52(7)^{\bullet \dagger}$ & $6.57 \pm 0.26(5)^{\bullet \dagger}$ \\
\hline VLFb & & NR (7) & NR (5) & NR (2) \\
\hline VLF-VC & & NR (6) & NR (6) & NR (6) \\
\hline $\mathrm{CV}$ & & NR (3) & NR (3) & NR (2) \\
\hline \multicolumn{5}{|c|}{ tcMMEP amplitude (mV) } \\
\hline $\mathrm{CV}$ saline control & & $8.88 \pm 2.06(4)^{\dagger}$ & $8.70 \pm 2.23(4)^{\dagger}$ & $6.15 \pm 2.05(2)^{\dagger \dagger}$ \\
\hline $\mathrm{VC}$ & $11.18 \pm 3.26(10)$ & $9.96 \pm 2.34(7)^{\dagger}$ & $11.99 \pm 3.13(7)^{\dagger}$ & $12.33 \pm 1.31(6)^{\dagger}$ \\
\hline VLFa & & $2.80 \pm 1.86(5)^{\bullet \dagger \dagger}$ & $2.71 \pm 2.70(7)^{\bullet \dagger \dagger}$ & $4.28 \pm 4.07(5)^{\bullet \dagger}$ \\
\hline $\mathrm{VLFb}$ & & NR (7) & NR (5) & NR (2) \\
\hline VLF-VC & & NR (6) & NR (6) & NR (6) \\
\hline $\mathrm{CV}$ & & NR (3) & NR (3) & NR (2) \\
\hline
\end{tabular}

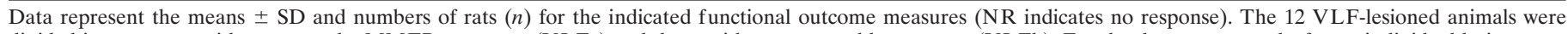

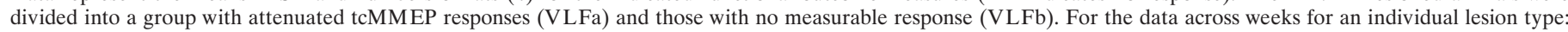

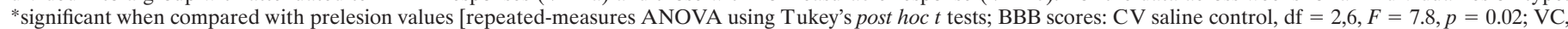

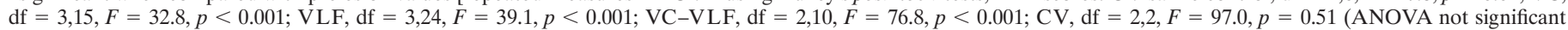

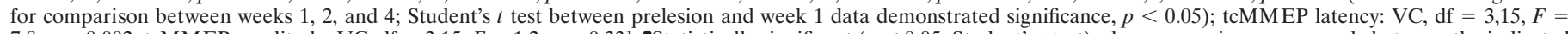

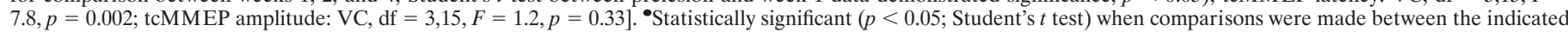

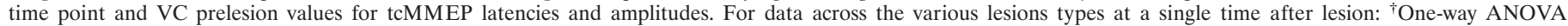

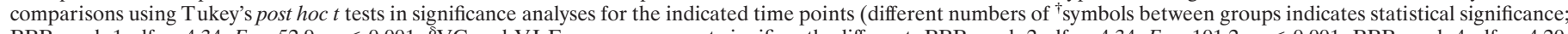

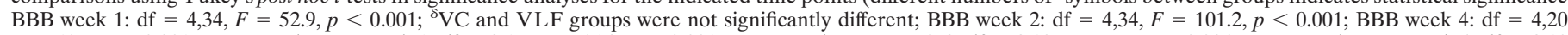

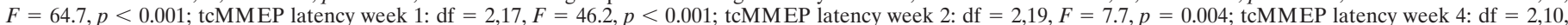

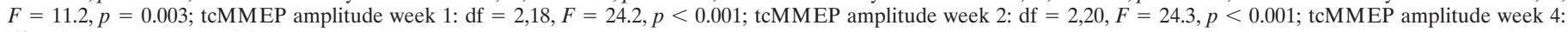
$\mathrm{df}=2,10, F=7.7, p=0.01$.

could be determined in the VLF animals exhibiting small steps or the VC animal that stepped laterally.

Figure 7 shows the number of hindlimb footfalls for pretrained animals walking on a grid both before lesion and after VC or VLF (VLFa and VLFb) lesion induction. Prelesion values were $0.1 \pm$ 0.1 and $0.3 \pm 0.1$ (footfalls per $150 \mathrm{~cm}$ trial) for VC and VLF groups, respectively. For postlesion induction, mean footfalls ranged from $0.5 \pm 0.4$ at 1 week to $0.3 \pm 0.6$ at 4 weeks for $\mathrm{VC}$ lesioned animals and $1.0 \pm 0.6$ to $1.0 \pm 1.1$ for VLF-lesioned animals. No statistical differences were revealed when comparisons were made over time (repeated-measures ANOVA) or between groups (paired $t$ tests). VLF-VC- and CV-lesioned animals did not exhibit locomotion sufficient to warrant grid-walking evaluation.

\section{DISCUSSION}

\section{Lesions}

This study examined the role(s) of ventral spinal cord pathways on open-field locomotion in the rat. Bilateral demyelinating VLF lesions at T9-T11, which completely blocked conduction in axons that transmit tcMMEPs, had little impact on BBB locomotor scores. Bilateral demyelinating VC lesions also produced only mild locomotor deficits. Thus, myelinated axons within either the VLF or VC are sufficient for the initiation of open-field locomotion in the rat. However, when VLF and VC lesions were combined or extended to encompass larger amounts of ventral white matter, severe locomotor deficits could be induced.

Historically, evidence supporting a role for the VLF in locomotion comes from studies demonstrating that MLR-evoked hindlimb fictive locomotion is lost after mechanical VLF lesioning (Steeves and Jordan, 1980) or selective VLF cooling (Noga et al., 1991). However, many have suggested that the MLRMedRF-VLF pathway does not represent a solitary system for the initiation of locomotion in the intact animal. For example, the VLF is not essential or necessary for partial recovery of hindlimb locomotion in birds (Sholomenko and Steeves, 1987), cats (Brustein and Rossignol, 1998), macaque monkeys (Vilensky et al., 1992), and man (Nathan, 1994).

In contrast to several other studies, our animals did not exhibit any significant recovery during the 4 week postlesion period. Commonly, a period of non-weight-supporting behavior is followed by a quick recovery (3-5 d) or functional improvements over an extended period (1-4 weeks). Rapid recovery may represent the return of function in spared reticulospinal fibers, whereas extended periods of recovery may involve complete lesioning of reticulospinal axons (Brustein and Rossignol, 1998) and a reorganization of descending signals for locomotion, possibly via a DLF pathway (Noga et al., 1991). Reorganization did not contribute significantly to our results because CV-lesioned animals displayed loss of weight support within hours after surgery that did not improve. The quick onset of paralysis was likely 
A.

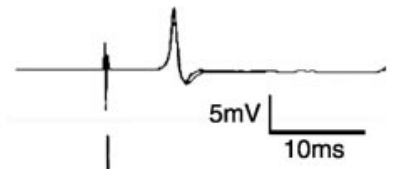

B.

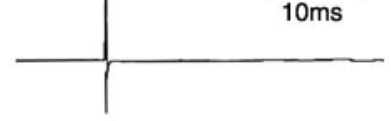

C.

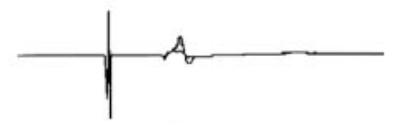

D.

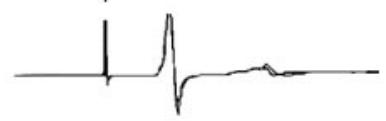

E.

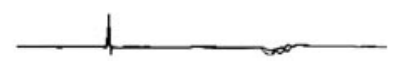

Figure 3. Representative examples of tcMMEP responses recorded from gastrocnemius muscles at 4 weeks after bilateral demyelinating lesions. $A$, $\mathrm{CV}$ saline control; $B, C, \mathrm{VLF} ; D, \mathrm{VC} ; E, \mathrm{VLF}-\mathrm{VC}$ or $\mathrm{CV}$. The difference between $B$ and $C$ is that $C$ is representative of VLF lesions that severely attenuated tcMMEP responses. The VLF was incompletely lesioned in these animals. tcMMEPs recorded from VC-lesioned animals did not differ significantly from saline controls.

the result of acute myelin sheath dysfunction secondary to oligodendrocyte intoxication with EB (Graca and Blakemore, 1986). With evidence of severe behavioral deficits acutely after the CV lesion, one would expect profound locomotor deficits within the same time period after VLF or VC lesions if either region contained myelinated axons necessary for activation of the CPG. No loss of weight support was ever observed in the VLF, VC, or VLF-VC groups.

These data suggest that, when demyelinating lesions are produced within the ventral rat spinal cord and the region is subsequently treated with irradiation to prevent remyelination,

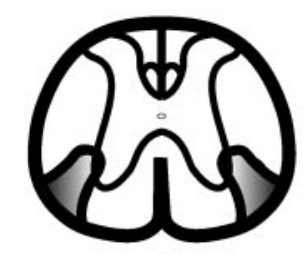

A

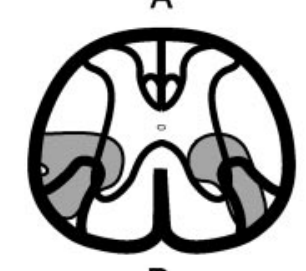

D

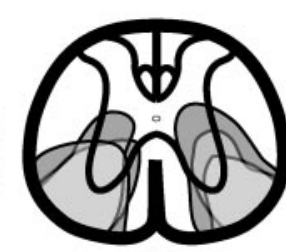

B

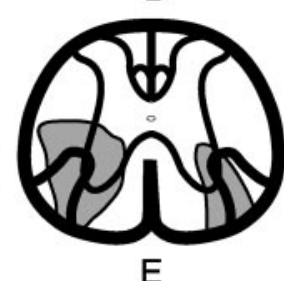

E

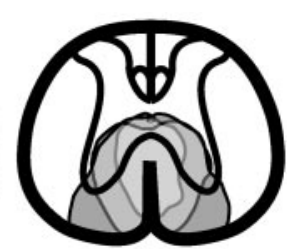

C

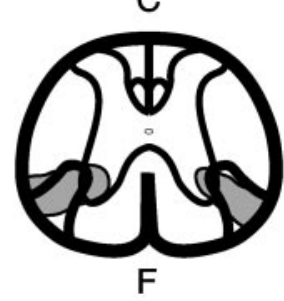

Figure 4. tcMMEP fiber strip located within the VLF. A, Sections from the epicenters of animals that showed diminished tcMMEP responses were overlaid with sections taken from the epicenters of lesions that did not significantly affect tcMMEP responses to delineate the minimal anatomical localization of the pathway that carries the tcMMEP. B, Extent of demyelination in three VLF-lesioned animals that exhibited complete loss of tcMMEPs at 4 weeks after lesion. Shading variations indicate areas of overlap between the three lesions. $C$, Extent of demyelination in three VC-lesioned animals that did not have significantly affected tcMMEP responses. $D-F$, Extent of demyelination in three VLF-lesioned animals in which tcMMEPs remained but were severely attenuated. In each rat, portions of the tcMMEP fiber strip $(A)$ were spared. reorganization sufficient to affect over-ground locomotor scores is not observed. Possible explanations include the following: (1) cues required to initiate reorganization may not be present in the demyelinated spinal cord in which axonal integrity is more widely preserved than in contusive injuries; and (2) subacute administration of high-dose irradiation $(>15 \mathrm{~Gy})$ may also be a factor in the observed lack of recovery because Ridet et al. (2000) have demonstrated detrimental effects on recovery after compressive SCI in rats. However, irradiation administered in high doses at $17 \mathrm{~d}$ after lesion (Kalderon and Fuks, 1996; Kalderon et al., 2001) or at low doses (2Gy) subacutely (Ridet et al., 2000) have been shown to stimulate axonal sprouting and facilitate recovery of function. High-dose irradiation characteristically prevents remyelination (Blakemore and Patterson, 1978); however, effects on other processes including axonal sprouting, glial scar formation, and angiogenesis, may subserve functionally significant roles.

The specificity of present lesions is also important because other studies have relied on surgical techniques that induce inconsistent damage to areas of the ventral spinal cord. These studies must be interpreted cautiously because results demonstrating correlation between lesioned regions of ventral spinal cord and locomotor deficits have not provided clear histological data that can be used for the design of targeted repair strategies.

Species differences may also play a role in the various results. Extensive ventral white matter lesions in the cat cause only moderate changes in locomotion (Brustein and Rossignol, 1998), whereas in birds (Sholomenko and Steeves, 1987) and rats (present data), similar lesions induce severe locomotor deficits or lasting paraplegia. Complete bilateral ventral hemisections in humans do not result in measurable locomotor deficits (Nathan, 1994). Collectively, these data suggest a considerable degree of interspecies variability in the functional redundancy of locomotor pathways.

Our lesions should not have interrupted action potential conduction in unmyelinated axons, in contrast to the previously used mechanical and selective cooling methods. Although the possibility that unmyelinated axons carried signals for activation of the CPG through the lesioned VLF or VC cannot be ruled out, the latencies of intraspinal field potentials recorded from the spinal cord in cats during MLR-evoked fictive locomotion are consistent with myelination (Noga et al., 1995). However, administration of noradrenergic and serotonergic drugs significantly enhances locomotor function after SCI, suggesting a significant role for unmyelinated ceruleospinal and raphespinal axons in the recovery of locomotor function (Barbeau et al., 1993; Barbeau and Rossignol, 1994; Chau et al., 1998a,b; Brustein and Rossignol, 1999). Based on previous data demonstrating the VLF as essential for MLRevoked fictive locomotion and the present data, it is likely that the MLR-MedRF-VLF pathway may represent a primary locomotor pathway within a comprehensive locomotor system capable of CPG activation via alternative pathways after VLF lesions.

When the VLF is evaluated in the context of a larger locomotor system, there are several explanations for the lack of locomotor deficits after discrete ventral cord lesions. Local interneuron cell columns, such as the propriospinal tract, may bypass the VLF lesions (Kazennikov et al., 1979; Budakova and Shik, 1980). However, functionally significant propriospinal contributions in this study, if present, must be diffusely 

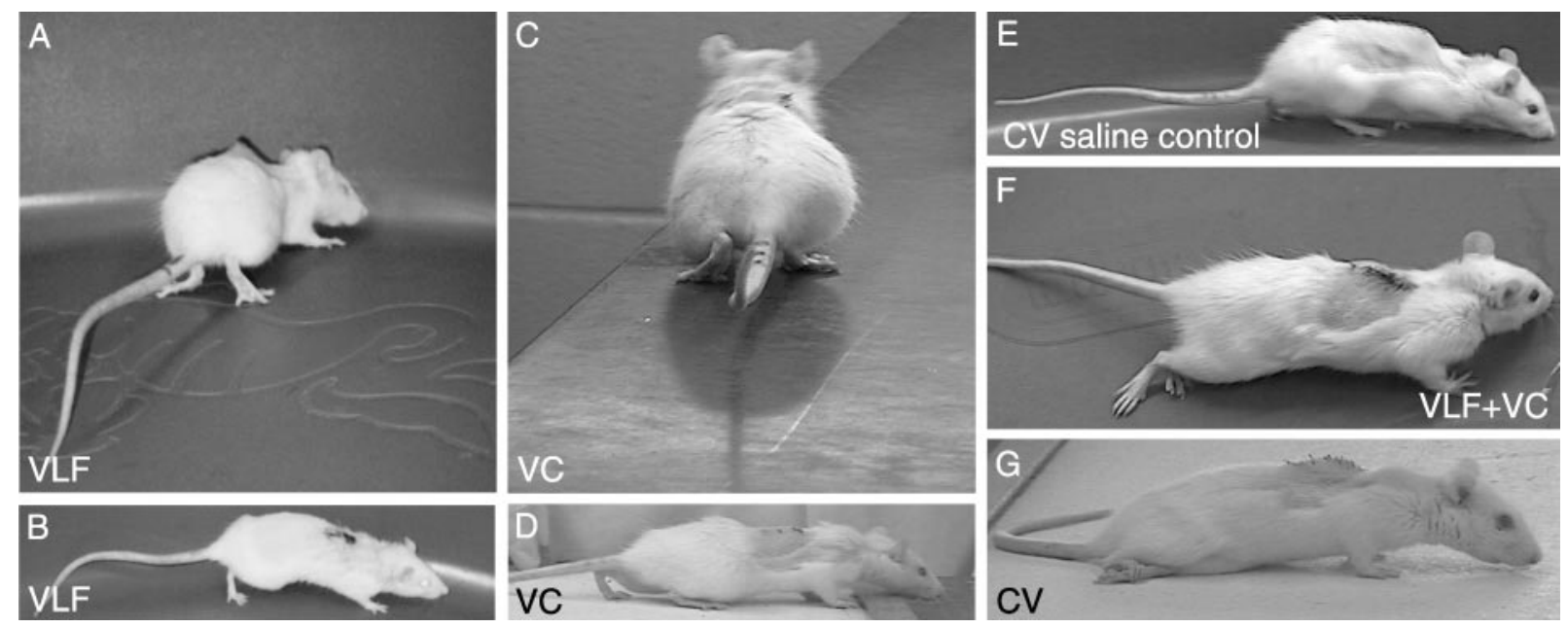

Figure 5. Open-field locomotion 4 weeks after various bilateral lesions within the thoracic ventral spinal cord. $A, B, \mathrm{VLF} ; C, D, \mathrm{VC} ; E$, control; $F$, VLF-VC; and $G, \mathrm{CV}$. In contrast to control animals $(E)$, note the prominent toe walking in VLF-lesioned animals $(A, B)$. $C, D$, VC lesions produced BBB scores similar to VLF lesions with less frequent toe walking. $F, \mathrm{VLF}-\mathrm{VC}$-lesioned animals displayed severe deficits in coordination but were always capable of weight support. $G, \mathrm{CV}$ lesions produced flaccid paralysis of the hindlimbs.

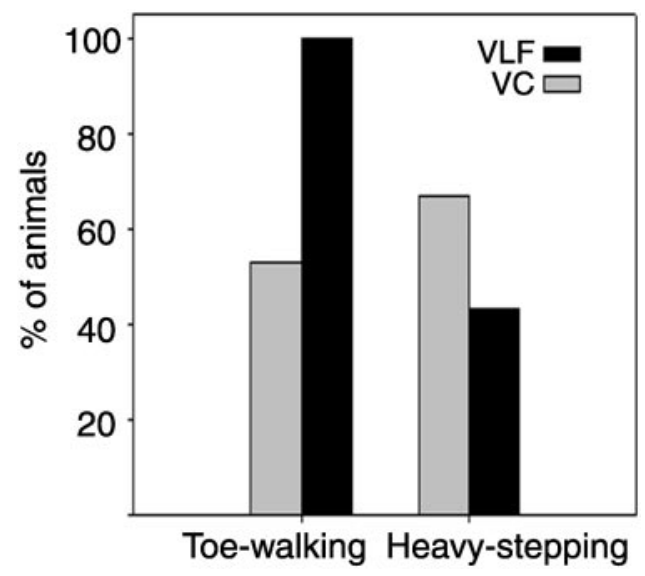

Figure 6. Locomotor deficits exhibited in VC- and VLF-lesioned animals not recognized by the BBB scoring system. All VLF-lesioned animals displayed toe walking during open-field locomotion, and half of VC-lesioned animals exhibited toe walking that was less qualitatively severe. Sixty-seven percent of VC-lesioned animals displayed heavy stepping compared with $43 \%$ of VLF-lesioned animals $(n=12$ and 7 for VLF and $\mathrm{VC}$, respectively).

arranged in the ventral half of the spinal cord (for review, see Ritter et al., 1999) because VC-VLF and CV lesions produced significant locomotor deficits and flaccid paralysis, respectively, without significant bilateral damage to dorsal horn propriospinal cells of origin. Despite the clear histologic confirmation afforded by the methods used here, each lesion type is a partial interruption of multiple pathways. Pathways likely involved in ventral spinal cord lesions include reticulospinal, raphespinal, ceruleospinal, vestibulospinal, and spinocerebellar. The current status of pathways, including PLR-DLF, ceruleospinal, and raphespinal projections, must be interpreted with caution because redundancy within the locomotor system may exist such that lesioning of the VLF may unmask roles for these pathways in spontaneous locomotion. If multiple tracts carry the signal to initiate locomotion simultaneously, selective lesioning of any one of these tracts would not be expected to

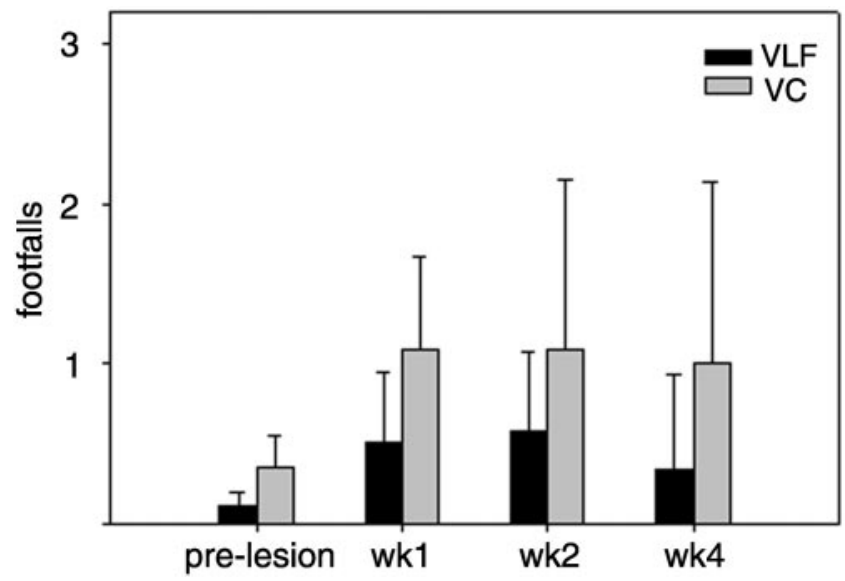

Figure 7. Grid-walking behaviors. Data represent the mean \pm SD of 12 and 7 animals for the VC and VLF lesions, respectively. Neither VC- nor VLF-lesioned animals displayed significant increases in footfalls while walking on the grid (VC, $\mathrm{df}=3,15 ; F=1.24 ; p=0.33$; V LF, $\mathrm{df}=3,24$; $F=1.008 ; p=0.42$ ).

remove input to the CPG. Alternatively, partial lesioning of one diff usely arranged tract, as may also be represented here, may not be sufficient to impart large locomotor deficits.

\section{tcMMEP fiber strip}

The tcMMEP has been used as a functional measurement of motor pathway damage in common models of SCI (Magnuson et al., 1999) and likely represents a bilateral activation of multiple subcortical structures with axons that descend in the VLF and synapse onto lumbar motoneurons monosynaptically. Previous studies demonstrated a monosynaptic pathway after stimulation of the surgically isolated VLF in neonatal rat spinal cord (Pinco and Lev-Tov, 1994). Although the location of this tcMMEP pathway also corresponds to the region proposed to carry fibers important for MLR-evoked locomotion (Steeves and Jordan, 1980; Jordan, 1986, 1991; Noga et al., 1991), stimulation of the MLR in cats elicited disynaptically and trisynaptically evoked field potentials in lumbar motor 
nuclei (Noga et al., 1995). Thus, although no conclusions can be made concerning any specific pathway represented by the tcMMEP signal, present evidence suggests that the tcMMEP does not represent a pathway involved in CPG activation. However, the tcMMEP will remain an important tool for the in vivo functional evaluation of SCI therapies as correlations with other voluntary movements are elucidated.

\section{Behavior}

VLF and VC lesions resulted in open-field locomotor deficits not fully indicated by the BBB scoring system. VLF- and VClesioned animals exhibited toe walking and heavy stepping, respectively. This behavior may represent an extensor bias derived from the lesioning of descending axons within the ventral white matter that act to modulate afferent hindlimb input onto lumbar spinal cord interneurons (Barbeau et al., 1999; Gosgnach et al., 2000). Neither VC- nor VLF-lesioned animals exhibited significant deficits on grid-walking tasks, a behavioral test chosen for its sensitivity in detecting hindlimb sensorimotor deficits (Behrmann et al., 1992). These data suggest that pathways within the lesioned areas of the VC and VLF do not contribute significantly to the sensorimotor control mechanisms needed to accomplish gridwalking tasks.

\section{Implications for spinal cord repair}

This study demonstrates that pathways within either the VLF or VC are sufficient for spontaneous open-field locomotion, despite the existence of a discrete fiber strip within the VLF that carries the tcMMEP. Fibers carrying the signals for the initiation of locomotion are likely redundant within the ventral half of the spinal cord because locomotor deficits are proportional to the amount of lesioned ventral white matter. Therapies aimed at the restoration of locomotor function in rat models of SCI should focus on generalized increases in functional white matter within the entire ventral half of the spinal cord because complete repair of all of these pathways may not be necessary.

\section{REFERENCES}

Barbeau H, Rossignol S (1994) Enhancement of locomotor recovery following spinal cord injury. Curr Opin Neurol 7:517-524.

Barbeau H, Chau C, Rossignol S (1993) Noradrenergic agonists and locomotor training affect locomotor recovery after cord transection in adult cats. Brain Res Bull 30:387-393.

Barbeau H, McCrea DA, O’Donovan MJ, Rossignol S, Grill WM, Lemay MA (1999) Tapping into spinal circuits to restore motor function. Brain Res Brain Res Rev 30:27-51.

Basso DM, Beattie MS, Bresnahan JC (1995) A sensitive and reliable locomotor rating scale for open field testing in rats. J Neurotrauma 12:1-21.

Basso DM, Beattie MS, Bresnahan JC (1996) Graded histological and locomotor outcomes after spinal cord contusion using the NYU weightdrop device versus transection. Exp Neurol 139:244-256.

Behrmann DL, Bresnahan JC, Beattie MS, Shah BR (1992) Spinal cord injury produced by consistent mechanical displacement of the cord in rats: behavioral and histologic analysis. J Neurotrauma 9:197-217.

Blakemore WF, Patterson RC (1978) Suppression of remyelination in the CNS by X-irradiation. Acta Neuropathol (Berl) 42:105-113.

Brustein E, Rossignol S (1998) Recovery of locomotion after ventral and ventrolateral spinal lesions in the cat. I. Deficits and adaptive mechanisms. J Neurophysiol 80:1245-1267.

Brustein E, Rossignol S (1999) Recovery of locomotion after ventral and ventrolateral spinal lesions in the cat. II. Effects of noradrenergic and serotoninergic drugs. J Neurophysiol 81:1513-1530.

Budakova NN, Shik ML (1980) Walking does not require continuity of the medullar "locomotion strip" (in Russian). Biull Eksp Biol Med 89:3-6.

Cao QL, Zhang YP, Howard RM, Walters WM, Tsoulfas P, Whittemore SR (2001) Pluripotent stem cells engrafted into the normal or lesioned adult rat spinal cord are restricted to a glial lineage. Exp Neurol 167:48-58.

Chau C, Barbeau H, Rossignol S (1998a) Early locomotor training with clonidine in spinal cats. J Neurophysiol 79:392-409.

Chau C, Barbeau H, Rossignol S (1998b) Effects of intrathecal alpha1and alpha2-noradrenergic agonists and norepinephrine on locomotion in chronic spinal cats. J Neurophysiol 79:2941-2963.

Garcia-Rill E, Skinner RD, Gilmore SA (1981) Pallidal projections to the mesencephalic locomotor region (MLR) in the cat. Am J Anat 161:311-321.

Gosgnach S, Quevedo J, Fedirchuk B, McCrea DA (2000) Depression of group Ia monosynaptic EPSPs in cat hindlimb motoneurones during fictive locomotion. J Physiol (Lond) 526:639-652.

Graca DL, Blakemore WF (1986) Delayed remyelination in rat spinal cord following ethidium bromide injection. Neuropathol Appl Neurobiol 12:593-605.

Honmou O, Felts PA, Waxman SG, Kocsis JD (1996) Restoration of normal conduction properties in demyelinated spinal cord axons in the adult rat by transplantation of exogenous Schwann cells. J Neurosci 16:3199-3208.

Imai T, Aoki M (1996) Temporal relationship between the recovery of longitudinal reflex systems and motor recovery in chronic spinallylesioned rats. Restor Neurol Neurosci 9:167-176.

Jeffery ND, Crang AJ, O'Leary MT, Hodge SJ, Blakemore WF (1999) Behavioural consequences of oligodendrocyte progenitor cell transplantation into experimental demyelinating lesions in the rat spinal cord. Eur J Neurosci 11:1508-1514.

Jordan LM (1986) Initiation of locomotion from the mammalian brainstem. In: Wenner-Gren Centre International Symposium Series, Vol 45, Neurobiology of vertebrate locomotion (Grillner S, Stein PSG, Stuart DG, Forssberg H, Herman RM, eds), pp 21-37. London: Macmillan.

Jordan LM (1991) Brainstem and spinal cord mechanisms for the initiation of locomotion. In: Neurobiological basis of human locomotion (Shimamura M, Grillner S, Edgerton VR, eds), pp 3-20. Tokyo: Japan Scientific Series.

Kalderon N, Fuks Z (1996) Structural recovery in lesioned adult mammalian spinal cord by $\mathrm{x}$-irradiation of the lesion site. Proc Natl Acad Sci USA 93:11179-11184.

Kalderon N, Xu S, Koutcher JA, Fuks Z (2001) Fractionated radiation facilitates repair and functional motor recovery after spinal cord transection in rat. Brain Res 904:199-207.

Kazennikov OV, Selionov VA, Shik ML, Iakovleva GV (1979) Neurons of the superior cervical segments responding to stimulation of the bulbar locomotor strip (in Russian). Neirofiziologiia 11:245-253.

Kazennikov OV, Shik ML, Iakovleva GV (1980) Two pathways for the "locomotor action" of the brain stem on the spinal cord (in Russian) Fiziol Zh SSSR IM I M Sechenova 66:1260-1263.

Linden RD, Zhang YP, Burke DA, Hunt MA, Harpring JE, Shields CB (1999) Magnetic motor evoked potential monitoring in the rat. J Neurosurg 91:205-210.

Magnuson DS, Trinder TC (1997) Locomotor rhythm evoked by ventrolateral funiculus stimulation in the neonatal rat spinal cord in vitro. J Neurophysiol 77:200-206.

Magnuson DS, Trinder TC, Zhang YP, Burke D, Morassutti DJ, Shields CB (1999) Comparing deficits following excitotoxic and contusion injuries in the thoracic and lumbar spinal cord of the adult rat. Exp Neurol 156:191-204.

Mori S, Shik ML, Yagodnitsyn AS (1977) Role of pontine tegmentum for locomotor control in mesencephalic cat. J Neurophysiol 40:284-295.

Mori S, Nishimura H, Kurakami C, Yamamura T, Aoki M (1978) Controlled locomotion in the mesencephalic cat: distribution of facilitatory and inhibitory regions within pontine tegmentum. J Neurophysiol 41:1580-1591.

Nathan PW (1994) Effects on movement of surgical incisions into the human spinal cord. Brain 117:337-346.

Noga BR, Kriellaars DJ, Jordan LM (1991) The effect of selective brainstem or spinal cord lesions on treadmill locomotion evoked by stimulation of the mesencephalic or pontomedullary locomotor regions. J Neurosci 11:1691-1700.

Noga BR, Fortier PA, Kriellaars DJ, Dai X, Detillieux GR, Jordan LM (1995) Field potential mapping of neurons in the lumbar spinal cord activated following stimulation of the mesencephalic locomotor region. J Neurosci 15:2203-2217.

Pinco M, Lev-Tov A (1994) Synaptic transmission between ventrolateral funiculus axons and lumbar motoneurons in the isolated spinal cord of the neonatal rat. J Neurophysiol 72:2406-2419.

Ridet JL, Pencalet P, Belcram M, Giraudeau B, Chastang C, Philippon J, Mallet J, Privat A, Schwartz L (2000) Effects of spinal cord X-irradiation on the recovery of paraplegic rats. Exp Neurol 161:1-14

Ritter A, Wenner P, Ho S, Whelan PJ, O'Donovan MJ (1999) Activity patterns and synaptic organization of ventrally located interneurons in the embryonic chick spinal cord. J Neurosci 19:3457-3471. 
Schwab ME, Brosamle C (1997) Regeneration of lesioned corticospinal tract fibers in the adult rat spinal cord under experimental conditions. Spinal Cord 35:469-473.

Selionov VA, Shik ML (1981) Responses of medulla oblongata neurons to microstimulation of the "locomotor strip" in cats (in Russian). Neirofiziologiia 13:275-282.

Shik ML, Iagodnitsyn AS (1977) Pontobulbar "locomotor strip" (in Russian). Neirofiziologiia 9:95-97.

Shik ML, Iagodnitsyn AS (1978) Reactions of cat hindbrain "locomotor strip" neurons to microstimulation (in Russian). Neirofiziologiia 10:510-518.

Shik ML, Severin FV, Orlovskii GN (1966) Control of walking and running by means of electric stimulation of the midbrain (in Russian). Biofizika 11:659-666.
Shik ML, Severin FV, Orlovskii GN (1967) Structures of the brain stem responsible for evoked locomotion (in Russian). Fiziol Zh SSSR IM I M Sechenova 53:1125-1132.

Sholomenko GN, Steeves JD (1987) Effects of selective spinal cord lesions on hind limb locomotion in birds. Exp Neurol 95:403-418.

Steeves JD, Jordan LM (1980) Localization of a descending pathway in the spinal cord which is necessary for controlled treadmill locomotion. Neurosci Lett 20:283-288.

Steeves JD, Jordan LM (1984) Autoradiographic demonstration of the projections from the mesencephalic locomotor region. Brain Res 307:263-276.

Vilensky JA, Moore AM, Eidelberg E, Walden JG (1992) Recovery of locomotion in monkeys with spinal cord lesions. J Motor Behav 24:288-296. 